\title{
Longitudinal changes in airway hyperresponsiveness and COPD mortality
}

\author{
To the Editor:
}

Airway hyperresponsiveness (AHR) is associated with an increased mortality risk among males [1] and chronic obstructive pulmonary disease (COPD) patients [2]. However, this association is largely based on cross-sectional studies using a single measurement of AHR to predict mortality; inherently ignoring the longitudinal variability of AHR. AHR is variable regardless of disease or medication status, and is linked with changes in smoking habits, seasonal variations and exposure to pollutants [3-5]. Considering this, it remains unclear how changes in AHR affect mortality, specifically from causes such as COPD, cardiovascular disease (CVD) and cancer.

We used data from the Vlagtwedde-Vlaardingen study (1965-1990) [6], a general population-based cohort of two communities in the Netherlands followed-up every 3 years. At all visits, AHR was measured using histamine biphosphate administered in increasing doses of $1,4,8,16$ and $32 \mathrm{mg} \cdot \mathrm{mL}^{-1}$ for $30 \mathrm{~s}$ followed by lung function measurements. The provocation concentration $\left(\mathrm{PC}_{10}\right)$ which caused a steady reduction in inspiratory vital capacity (IVC) or forced expiratory volume in $1 \mathrm{~s}\left(\mathrm{FEV}_{1}\right)$ of $>10 \%$ compared to baseline was then considered as the threshold value. Data on weight, height, eosinophil count, smoking habits and respiratory symptoms were also collected at each follow-up period. Vital status of all participants including specific causes of death was updated on December 31, 2016 at Statistics Netherlands (The Hague, the Netherlands). The causes of death were coded according to the International Classification of Diseases (versions 7-10).

We classified AHR based on histamine threshold, with $\leqslant 8 \mathrm{mg} \cdot \mathrm{mL}^{-1}$ indicating positive AHR (responders) and $>8 \mathrm{mg} \cdot \mathrm{mL}^{-1}$ indicating negative AHR (non-responders) [7]. We then included subjects who had a minimum of two AHR measurements and categorised changes in AHR from baseline (first available measurement) to the last available measurement for each subject as: "never AHR" (if subjects were non-hyperresponsive at baseline and during follow-up), "persistent AHR" (if subjects were hyperresponsive at baseline and during follow-up), "remitting AHR" (if subjects were hyperresponsive at baseline, but became non-hyperresponsive during follow-up), "developing AHR" (if subjects were non-hyperresponsive at baseline but became hyperresponsive during follow-up) and "variable AHR" (if subjects did not have a specific trend in AHR, constituting all remaining subjects). We calculated subject-specific annual change estimates for continuous variables (body mass index (BMI), FEV , FEV $_{1} /$ IVC ratio and eosinophil count) by taking the difference between the last and first available measurements and standardising this based on the number of years of follow-up. Changes in smoking and dyspnoea were used as defined previously $[8,9]$. Asthma attacks were self-reported attacks of shortness of breath with wheezing in any of the visits.

The association between changes in AHR and all-cause and cause-specific mortality was estimated using a Cox proportional-hazards model. The time to event was defined from the last available AHR measurement until the date of death or censoring on December 31,2016. We adjusted for lung function by including both baseline $\mathrm{FEV}_{1}$ and the yearly change estimate of $\mathrm{FEV}_{1}$ in the model. We also adjusted for other fixed (sex, place of residence, baseline age) and changing (BMI, eosinophil count, smoking status, dyspnoea and asthma attacks) covariates. For cause-specific mortality, subjects who died from causes other than the cause of interest were censored at the age of death and a competing risk analysis was performed [10]. Subjects who died due to external causes of death such as accidents and suicides were excluded from the analysis $(n=18)$.

In total, 1464 subjects had at least two AHR measurements. Among these, 702 (47.9\%) had died by the end of December 31, 2016 and only nine (0.6\%) were lost to follow-up (table 1). Among those who died,

@ERSpublications

Airway hyperresponsiveness displayed on multiple occasions independently leads to a higher risk of COPD death http://bit.ly/363Wfkg

Cite this article as: Teferra AA, Vonk JM, Boezen HM. Longitudinal changes in airway hyperresponsiveness and COPD mortality. Eur Respir J 2020; 55: 1901378 [https://doi.org/10.1183/ 13993003.01378-2019]. 
TABLE 1 Characteristics of the participants classified by changes in airway hyperresponsiveness (AHR)

\begin{tabular}{|c|c|c|c|c|c|}
\hline & Never AHR & Always AHR & Remitting AHR & Developing AHR & Variable AHR \\
\hline Male & $479(56.4)$ & $25(61.0)$ & $23(56.1)$ & $215(60.2)$ & $104(59.4)$ \\
\hline Baseline BMI $\mathbf{k g} \cdot \mathbf{m}^{-2}$ & $24.9 \pm 3.5$ & $26.4 \pm 4.1$ & $26.3 \pm 3.9$ & $25.6 \pm 3.3$ & $25.6 \pm 2.7$ \\
\hline Baseline $\mathrm{FEV}_{1} \mathrm{~L}$ & $3.38 \pm 0.75$ & $2.52 \pm 0.67$ & $2.67 \pm 0.80$ & $3.13 \pm 0.66$ & $3.08 \pm 0.76$ \\
\hline Baseline $\mathrm{FEV}_{1} / \mathrm{IVC} \%$ & $79.94 \pm 6.73$ & $67.57 \pm 11.61$ & $72.29 \pm 6.57$ & $76.32 \pm 7.63$ & $75.31 \pm 8.92$ \\
\hline Ex-smoker & $130(15.9)$ & $5(12.8)$ & $5(12.2)$ & $47(13.6)$ & $16(9.4)$ \\
\hline Current smoker & $415(50.8)$ & $26(66.7)$ & $24(58.5)$ & $205(59.4)$ & $101(59.1)$ \\
\hline Annual change in BMI $\mathrm{kg} \cdot \mathrm{m}^{-2}$ & $0.1 \pm 0.2$ & $0.1 \pm 0.2$ & $0.1 \pm 0.3$ & $0.1 \pm 0.2$ & $0.1 \pm 0.2$ \\
\hline Annual change in $\mathrm{FEV}_{1} \mathrm{~mL}^{-y_{e a r}}{ }^{-1}$ & $-19.3 \pm 39.2$ & $-34.7 \pm 31.0$ & $5.8 \pm 107.6$ & $-30.7 \pm 28.7$ & $-22.7 \pm 22.9$ \\
\hline Annual change in $\mathrm{FEV}_{1} / \mathrm{IVC} \%$-year ${ }^{-1}$ & $-0.22 \pm 0.70$ & $-0.49 \pm 0.88$ & $0.08 \pm 1.04$ & $-0.34 \pm 0.58$ & $-0.17 \pm 0.45$ \\
\hline \multicolumn{6}{|l|}{ Change in smoking status } \\
\hline Unstructured & $54(6.4)$ & $3(7.7)$ & $1(2.4)$ & $25(7.1)$ & $11(6.3)$ \\
\hline \multicolumn{6}{|l|}{ Vital status } \\
\hline Alive & $479(56.4)$ & $9(22.0)$ & $14(34.1)$ & $169(47.3)$ & $82(46.9)$ \\
\hline Dead & $365(42.9)$ & $32(78.0)$ & 27 (65.9) & $185(51.8)$ & 93 (53.1) \\
\hline Unknown & $6(0.7)$ & $0(0.0)$ & $0(0.0)$ & $3(0.8)$ & $0(0.0)$ \\
\hline \multicolumn{6}{|l|}{ Causes of death } \\
\hline COPD & 8 (2.2) & 6 (18.8) & $3(11.1)$ & $5(2.7)$ & $6(6.5)$ \\
\hline CVD & $120(32.9)$ & $11(34.4)$ & 9 (33.3) & $64(34.6)$ & 31 (33.3) \\
\hline Cancer & $139(38.1)$ & $6(18.8)$ & 9 (33.3) & $71(38.4)$ & 30 (32.3) \\
\hline Other & $98(26.8)$ & $9(28.1)$ & $6(22.2)$ & 45 (24.3) & $26(28.0)$ \\
\hline \multicolumn{6}{|l|}{ Hazard ratios $\#$} \\
\hline All & 1.00 & $0.96(0.63-1.46)$ & $0.95(0.62-1.45)$ & $0.95(0.78-1.17)$ & $1.18(0.91-1.51)$ \\
\hline
\end{tabular}

Data are presented as $\mathrm{n}(\%)$, mean $\pm \mathrm{SD}$ or hazard ratio $(95 \% \mathrm{CI})$. BMI: body mass index; $\mathrm{FEV}_{1}$ : forced expiratory volume in $1 \mathrm{~s}$; IVC: inspiratory vital capacity; COPD: chronic obstructive pulmonary disease; CVD: cardiovascular disease. \# : never AHR (referent).

28 (3.9\%) died of COPD, 235 (32.6\%) died of CVD and 255 (35.4\%) died due to cancer. Deceased subjects had a higher prevalence of AHR, were more often smokers and had lower lung function levels at baseline. Subjects who died of COPD had more AHR at baseline (35.7\%) than those who died of CVD (13.6\%) or cancer $(10.2 \%)$.

In the adjusted model, none of the four AHR-change categories, compared to never AHR, showed a statistically significant association with all-cause mortality. However, the adjusted cause-specific survival analysis showed a statistically significant association between changes in AHR and COPD mortality. Subjects with persistent AHR or variable AHR had a higher risk of dying of COPD compared to those who were never hyperresponsive. Specifically, throughout the observation period, subjects with persistent AHR were six times more likely to die of COPD (hazard ratio (HR) 6.07, 95\% CI 1.23-29.92) and those with variable AHR were four times more likely to die of COPD (HR 4.56, 95\% CI 1.2-17.24), both compared to those who never had AHR (table 1). We did not observe a significant association between changes in AHR and CVD or cancer mortality.

Cocḱroft and Davis [11] classified components of AHR as persistent and transient, reflecting airway remodelling and airway inflammation, respectively. In our study, subjects with persistent AHR probably had irreversible airway remodelling. Repetitive stimuli and a constant activation of the repair process are mechanisms behind airway remodelling in COPD leading to airway wall thickening, decrease in airway lumen size and reduced airflow $[12,13]$. These mechanisms can accelerate the progress of COPD and lead to premature death. Individuals with variable AHR probably present the transient component of AHR 
characterised by airway inflammation [11]. This independence of airway restructuring and airway inflammation in COPD [14] suggests potentially distinct pathophysiological mechanisms behind individuals with persistent AHR and variable AHR.

Clinical management of airway inflammation may possibly "normalise" AHR and lower long-term mortality risk from COPD, but future studies should be performed to investigate this. In our study, subjects with remitting AHR did not have a higher risk of COPD mortality compared to the never AHR. This suggests that "losing" AHR over time can have a favourable outcome on the risk of COPD death, and possibly indicates the gradual attenuation of airway inflammation in some individuals [15]. This improvement of AHR can also be related to lifestyle changes including smoking cessation and use of medications to manage asthmatic symptoms. Compared to those who died of CVD or cancer, subjects who died from COPD had a higher prevalence of asthmatic symptoms (wheezing and asthma attack) at baseline.

A major strength of our study is the extensive follow-up with minimal attrition and the use of objective and standardised measurements. People with low lung function levels $\left(\mathrm{FEV}_{1}<1.5 \mathrm{~L}\right)$ were excluded from AHR measurements. This is important to note since reduced $\mathrm{FEV}_{1}$ is related to mortality and $\mathrm{AHR}$, indicating that the results are best generalisable to those with good lung health. Although histamine provocation is no longer commonly used due to its adverse effects, it is very similar to a methacholine bronchoprovocation test [16]. A $\mathrm{PC}_{10} \leqslant 8 \mathrm{mg} \cdot \mathrm{mL}^{-1}$ in the 30 -s protocol used in our study is comparable to a $\mathrm{PC}_{20} \leqslant 4 \mathrm{mg} \cdot \mathrm{mL}^{-1}$ in the 2-min protocol, which is the recommended cut-point for positive AHR [17]. We were not able to control for other possible confounders such as medication status and use of inhaled corticosteroids (which are shown to influence both AHR and COPD mortality), clinical data on lung phenotypes, airway calibre, occupational exposure and ambient air pollution. Finally, the use of administrative data for outcome assessment might have introduced some misclassification bias on the causes of death.

With a longitudinal cohort accruing 51 years of follow-up, repeated AHR measurements and control for previously suggested confounders such as lung function and smoking [18], we show that AHR that is present at all occasions (persistent) or some occasions but not always (variable) is associated with a higher risk of COPD mortality. Early targeting of individuals who display AHR on multiple occasions or those with varying AHR measurements could be an important step in reducing COPD related deaths.

Andreas A. Teferra $\oplus^{1,2,3}$, Judith M. Vonk $\oplus^{1,2}$ and H. Marike Boezen ${ }^{1,2}$

${ }^{1}$ Department of Epidemiology, University of Groningen, University Medical Center Groningen, Groningen, The Netherlands. ${ }^{2}$ Groningen Research Institute for Asthma and COPD (GRIAC), University of Groningen, University Medical Center Groningen, Groningen, The Netherlands. ${ }^{3}$ Division of Epidemiology, College of Public Health, The Ohio State University, Columbus, OH, USA.

Correspondence: H. Marike Boezen, Department of Epidemiology, University of Groningen, University Medical Center Groningen, Hanzeplein 1, 9700 RB, Groningen, The Netherlands. E-mail: h.m.boezen@umcg.nl

Received: 11 July 2019 | Accepted after revision: 15 Oct 2019

Acknowledgements: The authors would like to thank Statistics Netherlands (CBS) for providing access to vital status data for the participants. We also want to thank Douwe Postmus and Hans Burgerhof (Dept of Epidemiology, University of Groningen, Groningen, the Netherlands) for providing valuable statistical advice.

Conflict of interest: A.A. Teferra has nothing to disclose. J.M. Vonk has nothing to disclose. H.M. Boezen has nothing to disclose

\section{References}

1 Becker EC, Wölke G, Heinrich J. Bronchial responsiveness, spirometry and mortality in a cohort of adults. J Asthma 2013; 50: 427-432.

2 Hospers JJ, Postma DS, Rijcken B, et al. Histamine airway hyper-responsiveness and mortality from chronic obstructive pulmonary disease: a cohort study. Lancet 2000; 356: 1313-1317.

3 Redline S, Tager IB, Speizer FE, et al. Longitudinal variability in airway responsiveness in a population-based sample of children and young adults. Intrinsic and extrinsic contributing factors. Am Rev Respir Dis 1989; 140: $172-178$.

4 Rijcken B, Schouten JP, Weiss ST, et al. Long-term variability of bronchial responsiveness to histamine in a random population sample of adults. Am Rev Respir Dis 1993; 148: 944-949.

5 Chinn S, Jarvis D, Luczynska CM, et al. An increase in bronchial responsiveness is associated with continuing or restarting smoking. Am J Respir Crit Care Med 2005; 172: 956-961.

6 Van der Lende R. Epidemiology of Chronic Non-specific Lung Disease (Chronic Bronchitis). Groningen, University of Groningen, 1969.

7 Xu X, Rijcken B, Schouten JP, et al. Airways responsiveness and development and remission of chronic respiratory symptoms in adults. Lancet 1997; 350: 1431-1434.

8 Taghizadeh N, Vonk JM, Boezen HM. Lifetime smoking history and cause-specific mortality in a cohort study with 43 years of follow-up. PLoS One 2016; 11: e0153310. 
9 Figarska SM, Boezen HM, Vonk JM. Dyspnea severity, changes in dyspnea status and mortality in the general population: the Vlagtwedde/Vlaardingen study. Eur J Epidemiol 2012; 27: 867-876.

10 Putter H, Fiocco M, Geskus RB. Tutorial in biostatistics: competing risks and multi-state models. Stat Med 2007; 26: 2389-2430.

11 Cockcroft DW, Davis BE. Mechanisms of airway hyperresponsiveness. J Allergy Clin Immunol 2006; 118: 551-559.

12 Hirota N, Martin JG. Mechanisms of airway remodeling. Chest 2013; 144: 1026-1032.

13 Ganesan S, Sajjan US. Repair and remodeling of airway epithelium after injury in chronic obstructive pulmonary disease. Curr Respir Care Rep 2013; 2: 145-154.

14 Lapperre TS, Snoeck-Stroband JB, Gosman MME, et al. Dissociation of lung function and airway inflammation in chronic obstructive pulmonary disease. Am J Respir Crit Care Med 2004; 170: 499-504.

15 Barnes N. Reducing inflammation in COPD: the evidence builds. Thorax 2007; 62: 927-928

16 Cockcroft D, Davis B. Direct and indirect challenges in the clinical assessment of asthma. Ann Allergy Asthma Immunol 2009; 103: 363-372. 400.

17 Crapo RO, Casaburi R, Coates AL, et al. Guidelines for methacholine and exercise challenge testing - 1999. Am J Respir Crit Care Med 2000; 161: 309-329.

18 Vestbo J, Hansen EF. Airway hyperresponsiveness and COPD mortality. Thorax 2001; 56: 11-14.

Copyright @ERS 2020 